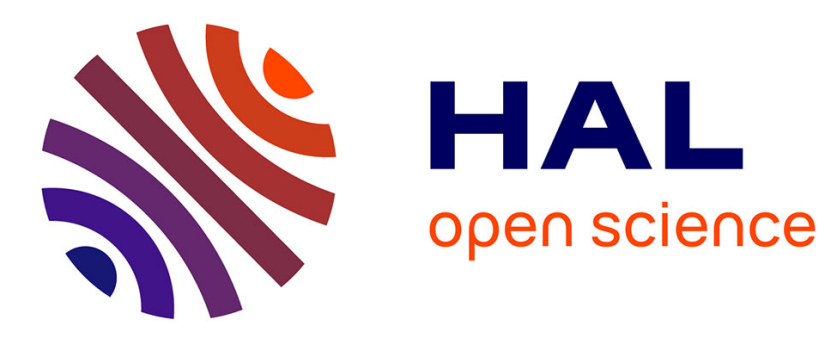

\title{
Numerical diffraction by an uniform grid
}

A. Bamberger, J.C. Guillot, Patrick Joly

\section{To cite this version:}

A. Bamberger, J.C. Guillot, Patrick Joly. Numerical diffraction by an uniform grid. RR-0472, INRIA. 1986. inria-00076082

\section{HAL Id: inria-00076082 \\ https://hal.inria.fr/inria-00076082}

Submitted on 24 May 2006

HAL is a multi-disciplinary open access archive for the deposit and dissemination of scientific research documents, whether they are published or not. The documents may come from teaching and research institutions in France or abroad, or from public or private research centers.
L'archive ouverte pluridisciplinaire HAL, est destinée au dépôt et à la diffusion de documents scientifiques de niveau recherche, publiés ou non, émanant des établissements d'enseignement et de recherche français ou étrangers, des laboratoires publics ou privés. 


\title{
NUMERICAL DIFFRACTION BY AN UNIFORM GRID
}

\author{
A. BAMBERGER ; J.C. GUILLOT and P. JOLY ${ }^{*}$.
}

* Centre de Mathématiques Appliquées de l'Ecole Polytechnique 91128 Palaiseau Cédex. France.

${ }^{\star *}$ I.N.R.I.A., Domaine de Voluceau - Rocquencourt 78153 Le Chesnay France. 


\section{RESUME}

Dans ce rapport, nous analysons l'influence $d^{\prime}$ une discrétisation en espace. sur la propagation $d^{\prime}$ une source ponctuelle harmonique.

Nous étudions, pour différents schémas d'approximation de l'équation des ondes par différences finies ou éléments finis sur des grilles régulières, le comportement asymptotique en espace de la solution élémentaire de l'équation de Helmholtz associée. Nous nous intéressons à la déformation des fronts d'onde et à la répartition d'amplitude le long de ces fronts. Nous quantifions l'anisotropie et la dispersion observées en fonction du nombre d'onde et du pas de discrétisation.

Nous mettons ainsi en évidence des résultats qui complètent ceux de l'analyse par ondes planes. Certains de ces résultats sont classiques en cristallographie.

La méthode d'analyse repose sur l'utilisation des techniques de phase stationnaire habituelles dans la théorie des équations aux dérivées partielles.

\section{ABSTRACT}

In this report we analyse the influence of a spatial discretization on the propagation of a wave generated by a harmonic point source.

We consider finite element and finite difference schemes on a uniform grid for the wave equation. We study the asymptotic behavior in space of the elementary solution of the associated. Helmholtz equation. We look at the shape of the wave fronts and at the distribution of the amplitude along these fronts. We measure the anisotropy and the dispersion as function of the wave number and the mesh size.

We derive results which complement those that have been obtained by plane wave analysis. Some of these results are classic in cristallography.

The method of analysis is based on stationnary phase techniques, commonly used in the theory of partial differential equations.

\section{MOTS-CLES}

Equation d'Helmholtz - Schéma numérique - Dispersion - Anisotropie - Phase stationnaire Comportement asymptotic.

\section{KEY WORDS}

Helmholtz equation - numeric scheme - Dispersion - Anisotropy - stationnary phase asymptotic behavior. 
- INTRODUCTION

In this article we analyze the influence of a spatial discretization on the propagation of a wave generated by a harmonic point source.

We consider finite element and finite difference schemes on an uniform grid for approximating the wave equation in $\mathbb{R}^{2}$. The point of view we follow in this article is to consider the outgoing Green function for the stationary problem. We then compute the asymptotic behaviour of the Green function. The wave front and the amplitude of the asymptotic wave completely describe the anisotropy and the dispersion of the chosen discretization of the Laplacian.

Let us note that the asymptotic wave front will not be homothetic to the wave surface as it is the case in the continuous problem. Thus for discretized media both the asymptotic wave front and the asymptotic amplitude are new characterizations of the anisotropy and dispersion of the schemes.

Our results are complementary to those which have been obtained by using plane wave analysis (see [1], [2], [3], [4], [5], [6]). Some of them are well known in crystallography.

Our analysis uses the method of stationary phase. An outline of this paper is as follows:

In section 2, we present the problem and give the numerical schemes we are going to study. We give the mathematical framework: and describe the principles of our analysis. The main results, theorems $2.1,2.2$ and 2.3 are given without proof (Detailed proofs can be found in [ ]). The results are general and valid for any of the numerical schemes we consider. Finally we introduce the concepts of discrete asymptotic wave fronts and discrete asymptotic amplitude which will be of use in section 3 for the comparison of the schemes.

In section 3, we describe more precisely the results obtained for each scheme. Different curves and diagrams are presented. Finaliy we compare the schemes by considering their properties with respect to anisotropy and dispersion. 
II - METHOD AND RESULTS

Before studying our problem let us first recall the well known results we can obtain for the Laplacian in $\mathbb{R}^{2}$.

II.1 - Helmholtz equation in $\mathbb{R}^{2}$

6

Let us consider a two dimensional homogeneous isotropic medium whose sound speed is equal to one: The acoustic wave $\phi$ corresponding to a harmonic source vibrating at a fixed frequency $\omega>0$ is supposed to satisfy the following equation

$$
\frac{d^{2} \phi}{d t^{2}}(x, t)-\Delta \phi(x, t)=f(x) e^{-i \omega t},(x, t) \in \mathbb{R}^{3}
$$

$f$ is a smooth function belonging to $\mathscr{S}\left(\mathbb{R}^{2}\right)$, the Schwartz space, which will be called the source function. Looking for a solution to (2.1) such that

$$
\phi(x, t)=u(x) e^{-i \omega t}, \quad(x, t) \in \mathbb{R}^{3}
$$

we find that $u$ must be a solution to the Helmholtz equation :

$$
-\Delta u-w^{2} u=f
$$

It is well known that (2.3) may have several solutions for $\omega>0$. In order to get an unique solution, one way consists of using the so called limiting absorption principle (cf. [8]). Indeed suppose that the medium is slightly absorbing. In this case, instead of (2.1) acoustic wave propagation is governed by the following equation :

$$
\frac{\partial^{\varepsilon} \phi_{\varepsilon}}{\partial t^{2}}(x, t)+\varepsilon \frac{\partial \phi \varepsilon}{\partial t}(x, t)-\Delta \phi_{\varepsilon}(x, t)=f(x) e^{-i \omega t}
$$

where $\varepsilon$ is supposed to be strictly positive. The solution $\phi_{\varepsilon}$ to $(2.4)$ is such that : 


$$
\phi_{\varepsilon}(x, t)=u_{\varepsilon}(x) e^{-i \omega t}
$$

where $u_{\varepsilon}(\cdot)$ is the solution to

$$
-\Delta u_{\varepsilon}-i \varepsilon \omega u_{\varepsilon}-\omega^{2} u_{\varepsilon}=f
$$

(2.6) has an unique solution $u_{\varepsilon}$ in $H^{2}\left(\mathbb{R}^{2}\right)$. One then shows that, when $\varepsilon$ tends to $o_{+}$, that $u_{\varepsilon}$ tends to $u$ in $H_{70 c}^{2}\left(\mathbb{R}^{2}\right)$ where $u$ is a solution to the Helmholtz equation (2.3). The function $u$ is called the outgoing solution to (2.3). This solution corresponds to the physical one associated with the source function $f$. It is the unique solution to (2.3) which satisfies the following Sommerfeld radiation condition at infinity :

$$
\frac{\partial u}{\partial r}(x)-i \omega u(x)=0\left(\frac{1}{r^{3 / 2}}\right)
$$

when $r \rightarrow+\infty$ with $r=|x|$.

Then we have

$$
u=G\left(\cdot, \omega^{2}+i 0\right) * f
$$

$G\left(., \omega^{2}+i 0\right)$ is the outgoing Green function :

$$
G\left(x, \omega^{2}+i 0\right)=\frac{i}{4} H_{0}^{(1)}(\omega r)
$$

One has :

$$
-\Delta G\left(., \omega^{2}+i 0\right)-\omega^{2} G\left(\cdot, \omega^{2}+i 0\right)=\delta
$$

where $\delta$ is the Dirac distribution at the origin. 
Suppose that $r$ tends to infinity. We then get from (2.9)

$$
G\left(x, \omega^{2}+i 0\right)=\frac{i}{(2 \pi)^{1 / 2}} \frac{1}{2(\omega r)^{1 / 2}} e^{i(\omega r-\pi / 4)}+0\left(r^{-3 / 2}\right)
$$

$$
G\left(x, t, \omega^{2}+i 0\right)=G\left(x, \omega^{2}+i 0\right) e^{-i \omega t} \text { is a solution to }(2.1) \text { corresponding }
$$
to $f=\delta$. It is a circular wave and its asymptotic behaviour for large $r$ is equal to :

$$
G\left(x, t, \omega^{2}+i 0\right) \sim \frac{i}{(2 \pi)^{1 / 2}} \frac{1}{2(\omega r)^{1 / 2}} e^{i \omega(r-t)} e^{-i \pi / 4} \cdot(r++\infty)
$$

Asymptotically $G\left(x, t, \omega^{2}+i 0\right)$ is an outgoing circular wave propagating with a speed equal to one and with a decreasing amplitude. Let us rewrite $(2.11)$ as

$$
G\left(x, t, \omega^{2}+i 0\right) \sim \frac{i}{(2 \pi)^{1 / 2}} \frac{A(\omega, \theta)}{r^{1 / 2}} e^{i r \vec{S}(\omega, \theta) \cdot \vec{\theta}} e^{-i \pi / 4} \cdot(r \rightarrow+\infty)
$$

where $(r, \theta)$ are the polar coordinates of $x$ and $\vec{\theta}$ the unit vector $(\cos \theta, \sin \theta)$ with

$$
\vec{S}(\omega, \theta)=\omega \vec{\theta}
$$

$$
A(\omega, \theta)=\frac{1}{2 \omega^{1 / 2}}
$$

(2.13) will be well adapted to the comparison with the discretized case. In that case $\vec{S}(\omega, \theta)$ will not be colinear to $\vec{\theta}$. and $A(\omega, \theta)$ will depend on $\vec{\theta}$. Let $\theta$ denote the extremity of the vector $\vec{\theta}$ whose the origin is 0 . For fixed $\omega$ and $R>0$ the asymptotic wave front is the set of points $r \vec{\theta}$ such that $r \vec{S}(\omega, \theta) \cdot \vec{\theta}=R^{2}$ for $\vec{\theta} \in S_{1}$ the unit circle in $\mathbb{R}^{2}$. 
The wave fronts are all homothetic. Two points belonging to the same wave front are in phase.

The continuous asymptotic amplitude is the set of points $A(\omega, \theta)$ for $\theta$ belonging to $S_{1}$. It characterizes the angular variation of the asymptotic behaviour of the solution along the wave front. Of course in the continuous case asymptotic wave fronts and asymptotic amplitudes are both circles with center 0 .

\section{II.2 - Discretized Helmholtz equations}

Let $h$ be a strictly positive number; $h$ is the step size of the following uniform grid

$$
\mathbb{R}_{h}^{2}=\left\{x \in \mathbb{R}^{2} ; x=(p h, q h),(p, q) \in Z^{2}\right\}
$$

Let $L_{h}^{2}$, denote the following set of square integrable complex sequences :

$$
L_{h}^{2}=\left\{v_{h}: \mathbb{R}_{h}^{2} \rightarrow \mathbb{C} ; \sum_{x \in \mathbb{R}_{h}^{2}}\left|v_{h}(x)\right|^{2}<\infty\right\}
$$

$L_{h}^{2}$ is a Hilbert space for the following scalar product

$$
\left(v_{h}, w_{h}\right)_{L_{h}^{2}}=h^{2} \sum_{x \in \mathbb{R}_{h}^{2}} v_{h}(x) \bar{w}_{h}(x)
$$

Let $F_{h}$ be the discrete Fourier transform defined on $L_{h}^{2}$

$$
\left(\mathscr{F}_{h} v_{h}\right)(k)=\hat{v}_{h}(k)=\frac{1}{2 \pi} 1 \cdot i \cdot m \cdot \sum_{x \in \mathbb{R}_{h}^{2}} v_{h}(x) e^{-j k \cdot x}
$$

where $k \in K_{h}=\left[-\frac{\pi}{h}, \frac{\pi}{h} j \times\left[-\frac{\pi}{h}, \frac{\pi}{h}\right]\right.$. 
$F_{h}$ is unitary from $L_{h}^{2}$ onto $L^{2}\left(K_{h}\right)$. Let $\mathscr{F}_{h}^{*}$ be the adjoint of $F_{h} \cdot \mathscr{F}_{h}^{*}$ is the inverse of $F_{h}$ and we have

$$
\left(F_{h}^{*} \hat{v}_{h}\right)(x)=\frac{1}{2 \pi} \lim \int_{K_{h}} \dot{v}_{h}(k) e^{i k \cdot x} d k, x \in \mathbb{R}_{h}^{2} .
$$

To the wave equation $(2.1)$ on $\mathbb{R}^{2}$ corresponds a semi discretized wave equation on $\mathbb{R}_{h}^{2}$

$$
\frac{d^{2} \phi_{h}}{d t^{2}}-\Delta_{h} \phi_{h}=f_{h} e^{-i \omega t} \quad t \in \mathbb{R}
$$

$\Delta_{h}$ is a discretized Laplacian and $f_{h}$ is an approximation of the source $f$. Looking for a solution to (2.19) of the form

$$
\phi_{h}=u_{h} e^{-i \omega t}
$$

where $u_{h}$ is a complex valued function defined on $\mathbb{R}_{h}^{2}$, we find that $u_{h}$ is a solution of an discretized Helmholtz equation

$$
-\Delta_{h} u_{h}-\omega^{2} u_{h}=f_{h}
$$

Let us suppose that $f_{h}($.$) is a rapidly decreasing sequence, i.e.$

$\forall n \in \mathbb{N}$,

$$
\sup _{(p ; q) \in \mathbb{Z}^{2}}\left(1+p^{2}+q^{2}\right)^{N / 2}\left|f_{h}(p h, q h)\right|<\infty
$$

We now describe the discretizations of the Laplacian which are considered in this article. For any complex valued function $v_{h}$ defined on $\mathbb{R}_{h}^{2}$, let $v_{p, q}$ denote $v_{h}(p h, q h)$. We then consider the following classical finite difference and finite elements schemes.

Scheme 1 (Five points finite difference scheme)

$$
-\left(\Delta_{h} v_{h}\right)_{p, q}=-\frac{v_{p+1, q^{-2 v} p, q+v} p-1, q}{h^{2}}-\frac{v_{p, q+1}-2 v_{p, q}+v_{p, q-1}}{h^{2}} .
$$


The order of this scheme is two. For every regular function $u(.,.) \in C^{\infty}\left(\mathbb{R}^{2}\right)$ one has

$$
-\left(\Delta_{h} u\right)(p h, q h)=-(\Delta u)(p h, q h)+\frac{h^{2}}{12}\left(\frac{\partial^{4} u}{\partial x_{1}^{4}}+\frac{\partial^{4} u}{\partial x_{2}^{4}}\right)(p h, q h)+0\left(h^{4}\right)
$$

Scheme 2 (Q1 Finite element scheme)

$$
\begin{aligned}
-\left(\Delta_{h} v_{h}\right)_{p, q}= & -\frac{1}{3} \frac{v_{p+1, q}+v_{p-1, q}+v_{p, q+1}+v_{p, q-1}-4 v_{p q}}{h^{2}}+ \\
& -\frac{2}{3} \frac{v_{p+1, q+1}+v_{p-1, q+1}+v_{p+1, q-1}+v_{p-1, q-1}-4 v_{p q}}{2 h^{2}} .
\end{aligned}
$$

The order of this scheme is two. For every regular function $u(.,$.$) .$ one has

$$
\begin{aligned}
-\left(\Delta_{h} u\right)(p h, q h)= & -(\Delta u)(p h, q h)+\frac{h^{2}}{12}\left(\frac{\partial^{4} u}{\partial x_{1}^{4}}+\frac{\partial^{4} u}{\partial x_{2}^{4}}+4 \frac{\partial^{4} u}{\partial x_{1}^{2} \partial x_{2}^{2}}\right)(p h, q h)+ \\
& +0\left(h^{4}\right)
\end{aligned}
$$

Scheme 3 (Arakawa's scheme)

$$
\begin{aligned}
-\left(\Delta_{h} v_{h}\right)_{p, q}= & -\frac{2}{3} \frac{v_{p+1, q}+v_{p-1, q}+v_{p, q+1}+v_{p, q-1}-4 v_{p, q}}{h^{2}}+ \\
& -\frac{1}{3} \frac{v_{p+1, q+1}+v_{p-1, q+1}+v_{p+1, q-1}+v_{p-1, q-1}-4 v_{p, q}}{2 h^{2}} .
\end{aligned}
$$

The order of this scheme is two. It is the only nine points approximation whose truncation error is isotropic at the first order. For a regular function $u(.,$.$) , we obtain$

$$
-\left(\Delta_{h} u\right)(p h, q h)=-(\Delta u)(p h, q h)-\frac{h^{2}}{24}\left(\Delta^{2} u\right)(p h, q h)+o\left(h^{4}\right) .
$$

Every discretized Laplacian $-\Delta_{h}$ is a bounded self adjoint operator in $L_{h}^{2}$. In fact we have for every $v_{h} \in L_{h}^{2}$ 


$$
\mathscr{F}_{h}\left(-\Delta_{h} v_{h}\right)(k)=D_{h}(k) \hat{v}_{h}(k) \quad \forall k \in K_{h}
$$

where,

$$
\text { Scheme 1: } D_{h}(k)=\frac{4}{h^{2}}\left(\sin ^{2} \frac{k_{1} h}{2}+\sin ^{2} \frac{k_{2} h}{2}\right)
$$

$$
\text { Scheme } 2: D_{h}(k)=\frac{1}{3 h^{2}}\left(8-2 \cos k_{1} h-2 \cos k_{2} h-4 \cos k_{1} h \cos k_{2} h\right)
$$$$
\text { Scheme } 3: D_{h}(k)=\frac{2}{3 h^{2}}\left(5-2 \cos k_{1} h-2 \cos k_{2} h-\cos k_{1} h \cos k_{2} h\right) \cdot
$$

The equation $\omega^{2}=D_{h}(k)$ is the dispersion relation of the scheme. When $|k| h$ is small, $\omega^{2}=D_{h}(k)$ must be an approximation to $\omega^{2}=|k|^{2}$, the dispersion relation for the wave equation. More precisely, we have :

$$
\begin{aligned}
& \text { Scheme 1: } D_{h}(k)=|k|^{2}-\frac{h^{2}}{12}\left(k_{1}^{4}+k_{2}^{4}\right)+|k|^{2} 0\left(|k|^{4} h^{4}\right) \\
& \text { Scheme 2: } D_{h}(k)=|k|^{2}-\frac{h^{2}}{12}\left(k_{1}^{4}+k_{2}^{4}+4 k_{1}^{2} k_{2}^{2}\right)+|k|^{2} 0\left(|k|^{4} h^{4}\right) \\
& \text { Scheme 3: } D_{h}(k)=|k|^{2}-\frac{h^{2}}{12}|k|^{4}+|k|^{2} 0\left(|k|^{4} h^{4}\right)
\end{aligned}
$$

(2.31)-(2.33) clearly show that the anisotropy of scheme \#3 will be less important than that of schemes \#1 and 2 . and we, have

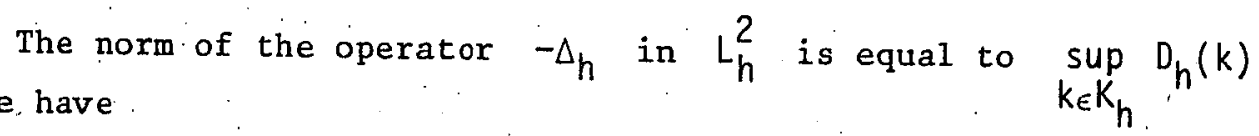

$$
\begin{aligned}
& \text { Scheme 1 }:\left\|\Delta_{h}\right\|=\frac{8}{h^{2}} \\
& \text { Scheme 2 }:\left\|\Delta_{h}\right\|=\frac{4}{h^{2}} \\
& \text { Scheme 3 }:\left\|\Delta_{h}\right\|=\frac{16}{3 h^{2}}
\end{aligned}
$$


Mimicking the continuous case we want to define the outgoing solution of the discretized Helmholtz equation $(2.20)$ by using the limiting absorption principle. Let us consider the discretized Helmholtz equation with some absorption in the same way as (2.6):

$$
-\omega^{2} u_{h}^{\varepsilon}-i \varepsilon \omega u_{h}^{\varepsilon}-\Delta_{h} u_{h}^{\varepsilon}=f_{h}, \quad(\varepsilon>0)
$$

Because of the selfadjointness of $-\Delta_{h}$ in $L_{h}^{2}$, the equation (2.34) has an unique solution $u_{h}^{\varepsilon}$ in $L_{h}^{2}$ for every $\varepsilon>0$. From the limiting absorption principle (cf [2]) we deduce that for every $(p, q) \in \mathbb{Z}^{2}$ and when $\omega^{2}$ is distinct from a finite set of exceptional values $u_{p, q}^{\varepsilon}$ tends to a limit $u_{p, q}$ as $\varepsilon$ tends to zero. This limit $u_{h}$ is a solution to the discretized Helmholtz equation and it is called the outgoing solution of $(2.20)$.

As in the continuous case we can express $u_{h}$ as a function of $f_{h}$ by using the so called outgoing Green function for the discretized Helmholtz equation.

Let $\delta_{h}$ be the discrete Dirac measure at the origin :

$$
\mid \begin{aligned}
& \delta_{0,0}=\frac{1}{h^{2}} \\
& \delta_{p, q}=0 \text { for }(p, q) \neq(0,0) .
\end{aligned}
$$

Note that $\delta_{h}$ belongs to $L_{h}^{2}$. Let $G_{h}^{\varepsilon}$ be the solution in $L_{h}^{2}$ to :

$$
-\omega^{2} G_{h}-i \omega \varepsilon G_{h}^{\varepsilon}-\Delta_{h} G_{h}^{\varepsilon}=\delta_{h} \text {. }
$$

We have :

$$
G_{h}^{\varepsilon}(x)=\frac{1}{2 \pi} \int_{K_{h}} \frac{e^{i k \cdot x}}{D_{h}(k)-\omega^{2}-i \omega \varepsilon} d k \quad, x \in \mathbb{R}_{h}^{2}
$$


As before from the limiting absorption principle we deduce that for every $(p, q) \in \mathbb{Z}^{2}$ and when $\omega^{2}$ is distinct from a finite set of values $G_{p, q}^{\varepsilon}$ tends to a limit $G_{p, q}$ as $\varepsilon$ tends to zero. This limit denoted by $G_{h}\left(\omega^{2}+i 0\right)$ is called the outgoing Green function for the discretized Helmholtz equation.

We have

$$
u_{h}=G_{h}\left(\omega^{2}+i 0\right) * f_{h}
$$

where

$$
u_{p, q}=h^{2} \sum_{\left(p^{\prime}, q^{\prime}\right) \in Z^{2}} G_{p^{\prime}, q^{\prime}}\left(\omega^{2}+i 0\right) f_{p-p^{\prime}, q-q^{\prime}} .
$$

Let. $\sigma\left(-\Delta_{h}\right)$ be the spectrum of $-\Delta_{h}$

$$
\sigma\left(-\Delta_{h}\right)=\left\{D_{h}(k) ; k \in K_{h}\right\} \text {. }
$$

Therefore we have :

$$
\begin{aligned}
& \text { Scheme 1: } \sigma\left(-\Delta_{h}\right)=\left[0, \frac{8}{h^{2}}\right] \\
& \text { Scheme 2: } \sigma\left(-\Delta_{h}\right)=\left[0, \frac{4}{h^{2}}\right] \\
& \text { Scheme 3: } \sigma\left(-\Delta_{h}\right)=\left[0, \frac{16}{3} \frac{1}{h^{2}}\right]
\end{aligned}
$$

Each operator $-\Delta_{h}$ is an absolutely continuous one. When $\omega^{2}$ does not belong to the spectrum $G_{h}\left(\omega^{2}+i 0\right)$ belongs to $L_{h}^{2}$ but when $\omega^{2}$ belongs to the spectrum of $-\Delta_{h} \quad G_{h}\left(\omega^{2}+i 0\right)$ does not belong to $L_{h}^{2}$. It is the objective of this article to give an expression of $G_{h}\left(\omega^{2}+i 0\right)$ and to study its asymptotic behaviour for large $|x|$ when $\omega^{2}$ belongs to the spectrum of $-\Delta_{h}$. Let $\Gamma_{h}$ be the following curve 


$$
\Gamma_{h}(\omega)=\left\{k \in K_{h} ; D_{h}(k)-\omega^{2}=0\right\}
$$

$\Gamma_{h}(\omega)$ is called a slowness surface. Let $\sigma_{c}\left(-\Delta_{h}\right)$ be the following set :

$$
\text { Scheme } 1: \sigma_{c}\left(-\Delta_{h}\right)=\{0\} \cup\left\{\frac{4}{h^{2}}\right\} \cup\left\{\frac{8}{h^{2}}\right\}
$$$$
\text { Scheme } 2: \sigma_{c}\left(-\Delta_{h}\right)=\{0\} \cup\left\{\frac{8}{3} \frac{1}{h^{2}}\right\} \cup\left\{\frac{3}{h^{2}}\right\} \cup\left\{\frac{4}{h^{2}}\right\}
$$

$$
\text { Scheme } 3: \sigma_{c}\left(-\Delta_{h}\right)=\{0\} \cup\left\{\frac{4}{h^{2}}\right\} \cup\left\{\frac{25}{6} \frac{1}{h^{2}}\right\} \cup\left\{\frac{16}{3} \frac{1}{h^{2}}\right\}
$$

on $\Gamma_{h}(\omega) \cdot \nabla D_{h}(k)$ is different from zero except when $\omega^{2}$ belongs to $\sigma_{c}\left(-\Delta_{h}\right)$.

Following the classical approach (cf. [9], [10], [11], [12], [13], [14], [15]) it is possible to consider the limit of (2.36) as $\varepsilon$ tends to zero and to get an expression for $G_{h}\left(\omega^{2}+j 0\right)$. For $x=(p h, q h)$ we set $G_{h}\left(\omega^{2}+i_{0}, x\right)$ instead of $G_{p, q}\left(\omega^{2}+i_{0}\right)$.

\section{THEOREM 2.1}

For every $\omega^{2}$ in $\sigma\left(-\Delta_{h}\right)-\sigma_{c}\left(-\Delta_{h}\right)$ and for every $x$ in $\mathbb{R}_{h}^{2} \quad G_{h}^{\varepsilon}(x)$ tends to $G_{h}\left(\omega^{2}+i 0, x\right)$ as $\varepsilon$ tends to zero. We have:

$$
\begin{aligned}
G_{h}\left(\omega^{2}+i 0, x\right) & =\frac{1}{2 \pi} P \cdot V \cdot \int_{K_{h} \cdot D_{h}(k)-\omega^{2}} \frac{1}{i k \cdot x} d k+ \\
& +\frac{i}{2} \int_{\Gamma_{h}(\omega)} \frac{1}{\nabla D_{h}(k) T} e^{i k \cdot x} d \gamma(k)
\end{aligned}
$$

where $\mathrm{dr}(k)$ is the measure induced on $\Gamma_{h}(\omega)$ by the Lebesgue one. $G_{h}\left(\omega^{2}+i o, x\right)$ is a solution to the discretized Helmholtz equation

$$
-\omega^{2} G_{h}-\Delta_{h} G_{h}=\delta_{h}
$$


Let us recall that if :

$$
0_{\delta}=\left\{k \in K_{h} \mid \operatorname{dist}\left(\Gamma_{h}(\omega), k\right)<\delta\right\}
$$

for $\delta$ small enough, we then have by definition of the principle value value

$$
P . V \int_{K_{h}} \frac{1}{D_{h}(k)-\omega^{2}} e^{i k \cdot x_{d k}}=\lim _{\delta \rightarrow 0} \int_{K_{h}-0_{\delta}} \frac{e^{i k \cdot x}}{D_{h}(k)-\omega^{2}} d k
$$

From (2.45) it is clear that $\omega^{2}$ cannot belong to $\sigma_{c}\left(-\Delta_{n}\right)$ because in that case $\nabla D_{h}(k)$ vanishes on $\Gamma_{h}(\omega)$. The expression (2.45) for $G_{h}\left(\omega^{2}+i 0\right)$ is well adapted to the study of the asymptotic behaviour of $G_{h}\left(\omega^{2}+i 0, x\right)$ when $|x|$ tends to infinity.

But for numerical purpose it is better to use an another expression for $G_{h}\left(\omega^{2}+i 0\right)$ given by the following theorem for the scheme \#1.

$\underline{T H E O R E M ~} 2.2$

In the case of the scheme 1 for $\omega^{2}$ in $\sigma\left(-\Delta_{h}\right)-\sigma_{c}\left(-\Delta_{h}\right)$ one has

$$
G_{p, q}\left(\omega^{2}+i o\right)=-\frac{1}{4 \pi} \int_{-M}^{+M} \frac{(r(\alpha))^{\dot{p}}}{b(\alpha)+r(\alpha)} e^{i q \alpha} d \alpha,
$$

with

$$
\begin{aligned}
& b(\alpha)=\cos \alpha-2+\frac{\omega^{2} h^{2}}{2} \\
& r(\alpha)=-b(\alpha)+i\left(1-(b(\alpha))^{2}\right)^{1 / 2} \text { if }|b(\alpha)|<1 \\
& r(\alpha)=-b(\alpha)+(\operatorname{sign}(b(\alpha)))\left((b(\alpha))^{2}-1\right)^{1 / 2} \text { if }|b(\alpha)| \geq 1 .
\end{aligned}
$$


Detailed proofs of theorems 2.1 and 2.2 can be found in [16]. (2.45) enables us to study the asymptotic behaviour of $G_{h}\left(\omega^{2}+i 0, x\right)$ by the method of stationary phase (cf. [12], [17]). Let us describe the different steps of the: calculation :

For every $x$ in $\mathbb{R}_{h}^{2}$ distinct from zero, one has $x=\vec{r}$ with $r=|x|$ and $\vec{\theta}=\frac{x}{r}$. $\vec{\theta}$ being fixed we study the asymptotic behaviour of $G_{h}\left(\omega^{2}+i 0\right)$ when $r$ tends to infinity. First one shows that

behaves as

$$
\frac{1}{2 \pi} P . V \int_{K_{h}} \frac{1}{D_{h}(k)-\omega^{2}} e^{i k \cdot x} d k
$$

$$
\frac{i}{2} s_{\Gamma_{h}(\omega)} \frac{e^{i k \cdot x}}{\nabla D_{h}(k) T}\left(\psi_{\theta}^{+}(k)-\psi_{\theta}^{-}(k)\right) d \gamma(k),
$$

as $r$ tends to infinity where $\psi_{\theta}^{+}(\cdot)$ (resp. $\psi_{\theta}^{-}(\cdot)$ ) is a smooth function on $\Gamma_{h}(\omega)$ which is equal to 1 and different from zero in a small neighbourhoud of the points for which the exterior normal to $\Gamma_{h}(\omega)$ is equal to $\vec{\theta}$ (resp. - $\vec{\theta}) \cdot G_{h}\left(\omega^{2}+i 0, x\right)$, given by $(2.45)$, behaves as

$$
\text { i } \delta_{\Gamma_{h}(\omega)} \frac{e^{i k \cdot x}}{\left|\nabla D_{h}(k)\right|} \psi_{\theta}^{+}(k) d_{\gamma}(k) \text {. }
$$

We then apply the method of stationary phase to the oscillating integral $\int_{\Gamma_{h}}(\omega)$ (cf. [8]) and we get the following result

\section{THEOREM 2.3}

such that

$$
\text { Let } g_{h}(\omega, \theta) \text { be the set of points } S_{h}(\omega ; \theta) \text { on } \Gamma_{h}(\omega)
$$

$$
\nabla D_{h}\left(S_{h}(\dot{\omega}, \theta)\right) /\left|\nabla D_{h}\left(S_{h}(\omega, \theta)\right)\right|=\vec{\theta}
$$

For the schemes we are considering $g_{h}(\omega, \theta)$ is always a finite set and $c_{h}\left(\omega, S_{h}(\omega, \theta)\right)$, the Gauss curvature at point $s_{h}(\omega, \theta)$ in $g_{h}(\omega, \theta)$, does not vanish. For. $S_{h}(\omega, \theta)$ in $g_{h}(\omega, \theta)$ the phase of 
the oscizlating integral $\int_{\Gamma_{h}(\omega)} \frac{e^{i k \cdot x}}{\nabla D_{h}(k) T} \psi_{\theta}^{+}(k) d \gamma(k)$ is stationary and we have

$$
\begin{aligned}
& G_{h}\left(\omega^{2}+i 0, r, \vec{\theta}\right)=\frac{1}{(2 \pi)^{1 / 2}} \sum_{S_{h}(\omega, \theta) \in g_{h}(\omega, \theta)} \frac{A_{h}(\omega, \theta)}{r^{1 / 2}} \\
& e^{i r \vec{S}_{h}(\omega, \theta) \cdot \vec{\theta}} e^{-i \pi / 4}+0\left(r^{-3 / 2}\right)
\end{aligned}
$$

when $r \rightarrow+\infty$.

$0($.$) is uniform with respect to \vec{\theta}$ and

$$
A_{h}(\omega, \theta)=\left|\nabla D_{h}\left(S_{h}(\omega, \theta)\right)\right|^{-1}\left|c_{h}\left(\omega, S_{h}(\omega, \theta)\right)\right|^{-1 / 2} .
$$

Each term of the asymptotic behaviour of $G_{h}\left(\omega^{2}+i o, r, \vec{\theta}\right)$ is characterized by its phase $\vec{S}_{h}(\omega, \theta)$. $\vec{\theta}$ and its amplitude $A_{h}(\omega, \theta)$ They both depend on $\vec{\theta}$ and their variation with respect to $\vec{\theta}$ characterizes the anisotropy of the scheme. Phases and amplitudes are functions of the product wh. The anisotropy of the scheme is much less important when wh $\sim 0$ than when wh $\sim 1$. We now define the discrete wave front and the discrete asymptotic amplitude as in section 2.1 .

$\omega^{2} \in \sigma\left(-\Delta_{h}\right)-\sigma_{c}\left(-\Delta_{h}\right)$ and $R>0$ being fixed the discrete asymptotic wave front associated with $(\omega, R)$ is the set of points $r \theta$ such that

$$
r \vec{S}_{h}(\omega, \theta) \cdot \vec{\theta}=R^{2}
$$

and the discrete asymptotic amplitude is the set of points $\left\{A_{h}(\omega, \theta) \theta ; \vec{\theta} \in S_{1}\right\}$.

Let us consider $G_{h}\left(\omega^{2}+i o, x\right) e^{-i \omega t}$ for each point of the grid. This function behaves, for fixed $\theta$ and large $r$, as an harmonic wave propagating with a phase speed equal to : 
(2.49)

$$
c_{\infty}^{h}(\omega, \theta)=\frac{\omega}{\vec{S}_{h}(\omega, \theta) \cdot \vec{\theta}}
$$

$c_{\infty}^{h}(\omega, \theta)$ is the discrete asymptotic speed in the direction $\vec{\theta}$. 


\section{III - DISCUSSION OF THE RESULTS CONCERNING THE SCHEMES}

\section{III.1 - Slowness surfaces for the schemes}

The basic tool for studying each scheme is the corresponding plot of the curves $\Gamma_{h}(\dot{w})$. Let us describe them (see [16] for detailed geometrical properties of these curves).

- Scheme 1

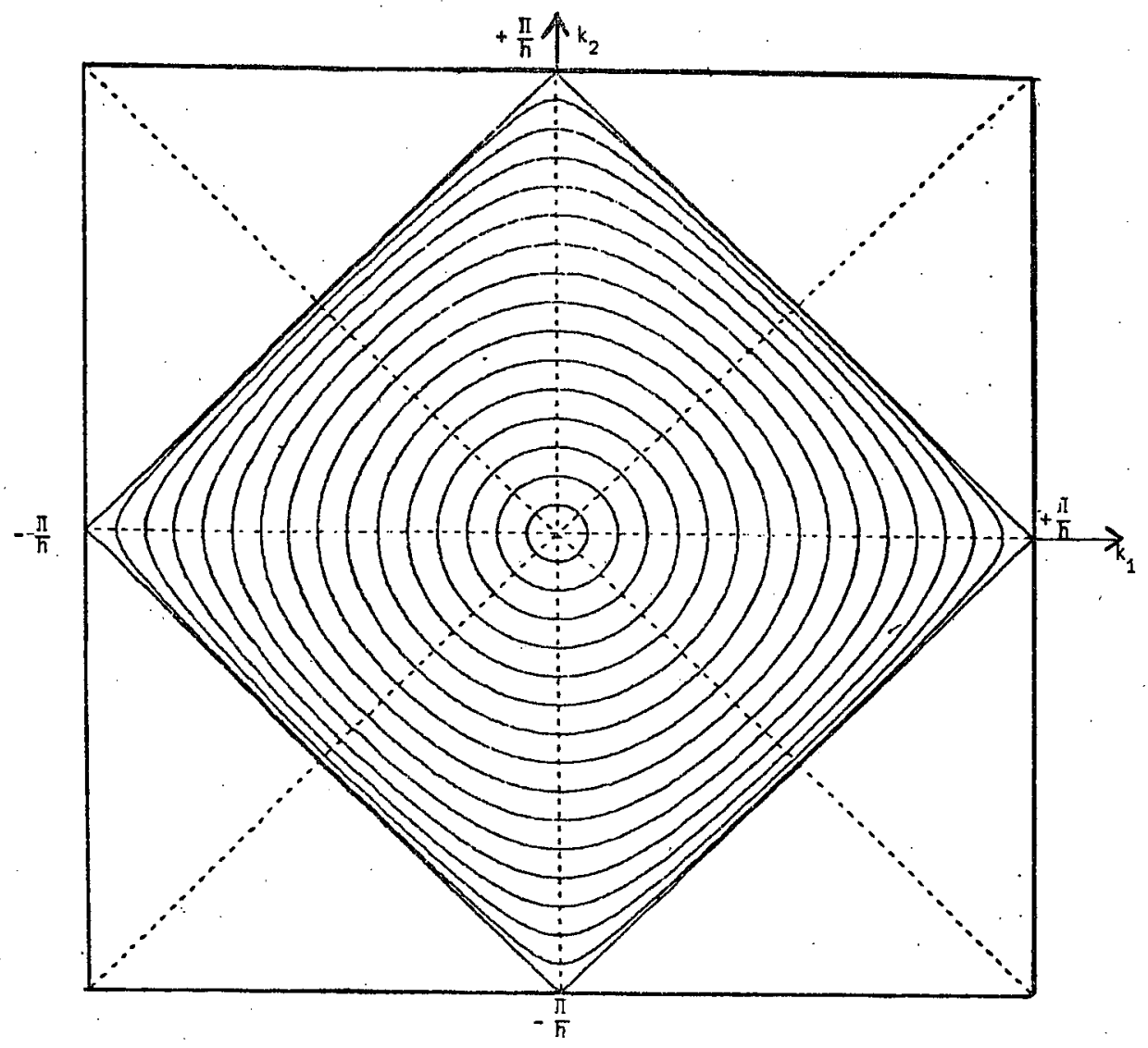

Figure 3.1

Scheme 1 : The curves $\Gamma_{h}(\omega)$ for $0<\omega^{2}<\frac{4}{h^{2}}$

For wh $\sim 0, \Gamma_{h}(\omega)$ is close to a circle and for $\omega h \sim 2, \Gamma_{h}(\omega)$ is close to a square (see Figure 3.1). 


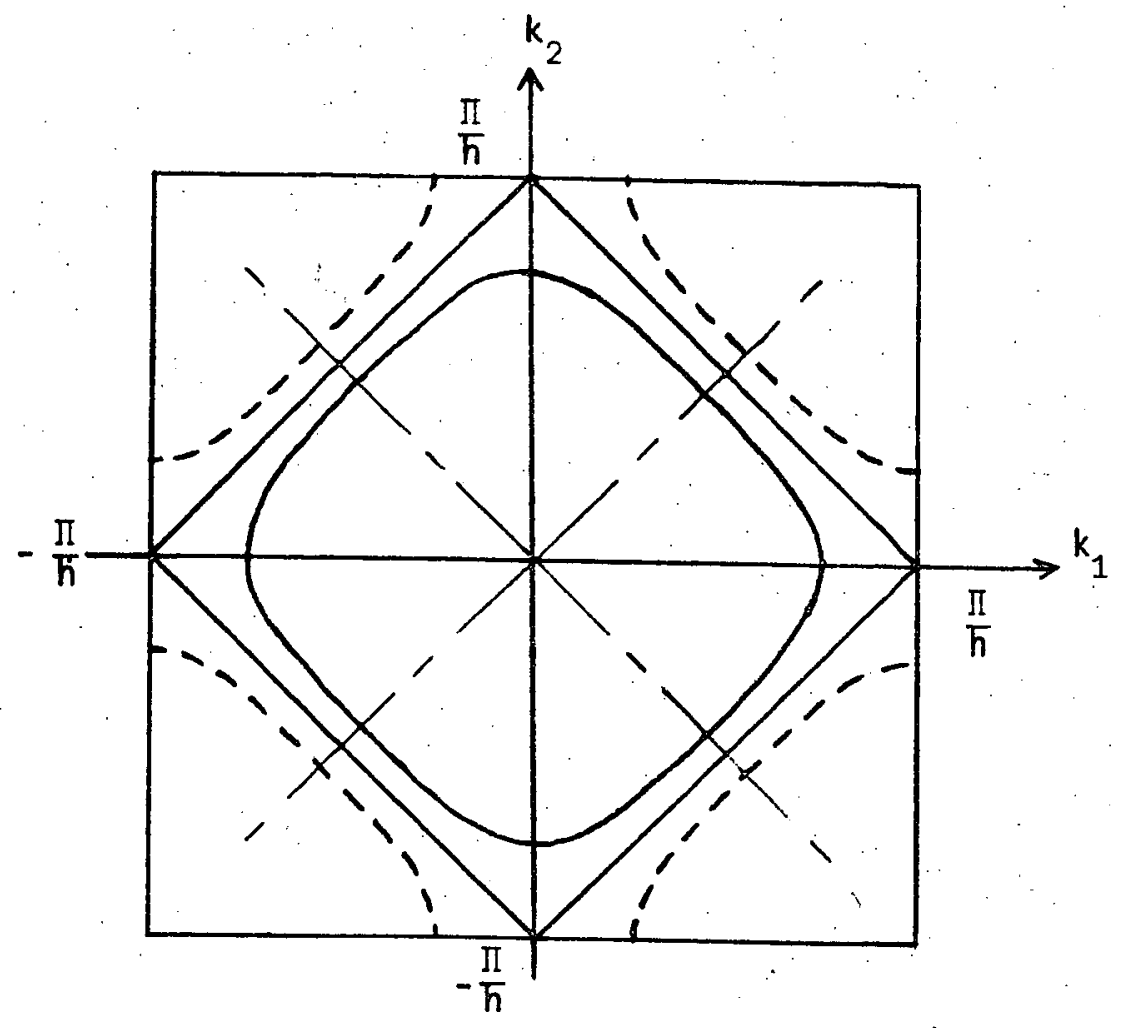

Figure 3.2

Scheme 1 : The curves $\Gamma_{h}(\omega)$ for $\frac{4}{h^{2}}<\omega^{2}<\frac{8}{h^{2}}$

For $\omega$ and $\omega^{\prime}$ such that $\omega^{2}+\left(\omega^{\prime}\right)^{2}=\frac{8}{h^{2}}, \Gamma_{h}\left(\omega^{\prime}\right)$ is, in the square $\left[0, \frac{\pi}{h}\right] \times\left[0, \frac{\pi}{h}\right]$, the symmetrical curve of $\Gamma_{h}(\omega)$ with respect to the point $\left(\frac{\pi}{2 h}, \frac{\pi}{2 h}\right)$. 
- Scheme 2

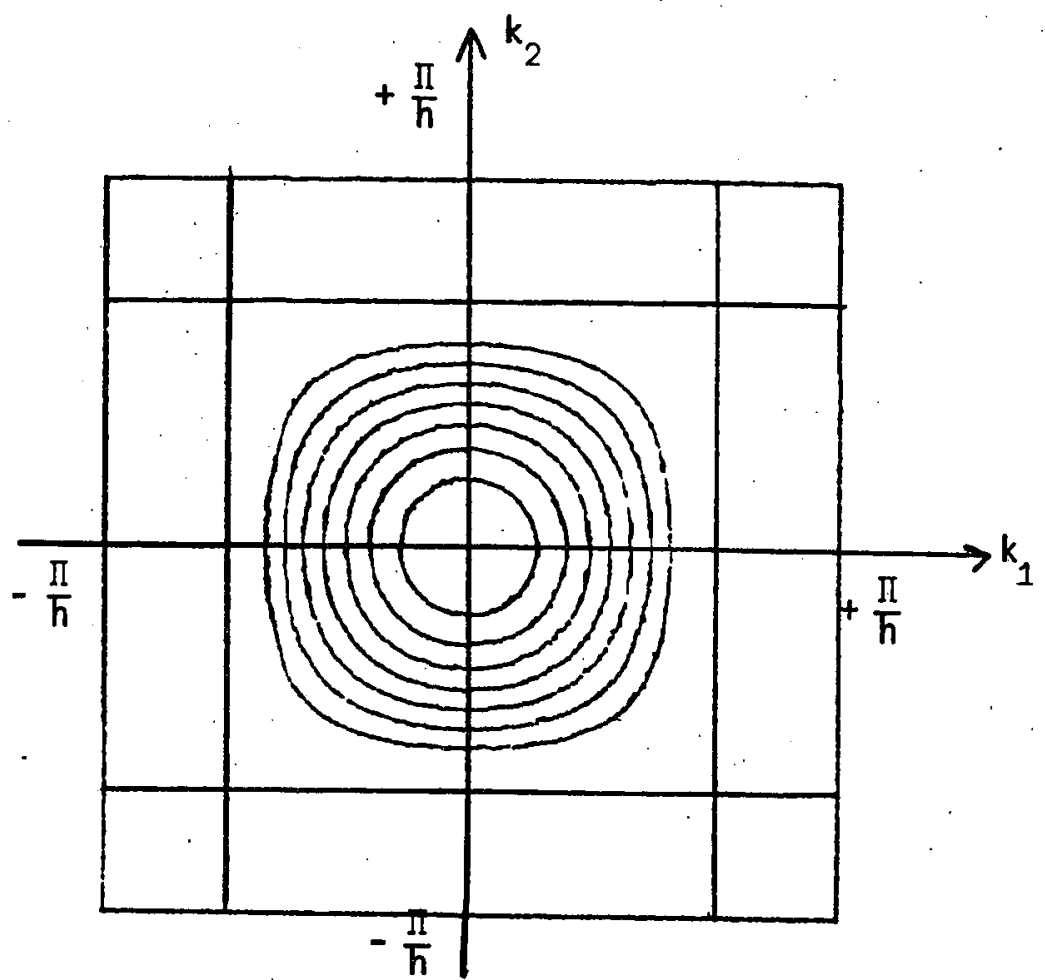

Figure 3.3

Scheme 2 : The curves $\Gamma_{h}(\omega)$ for $0<\omega^{2} h^{2}<\frac{8}{3}$ 


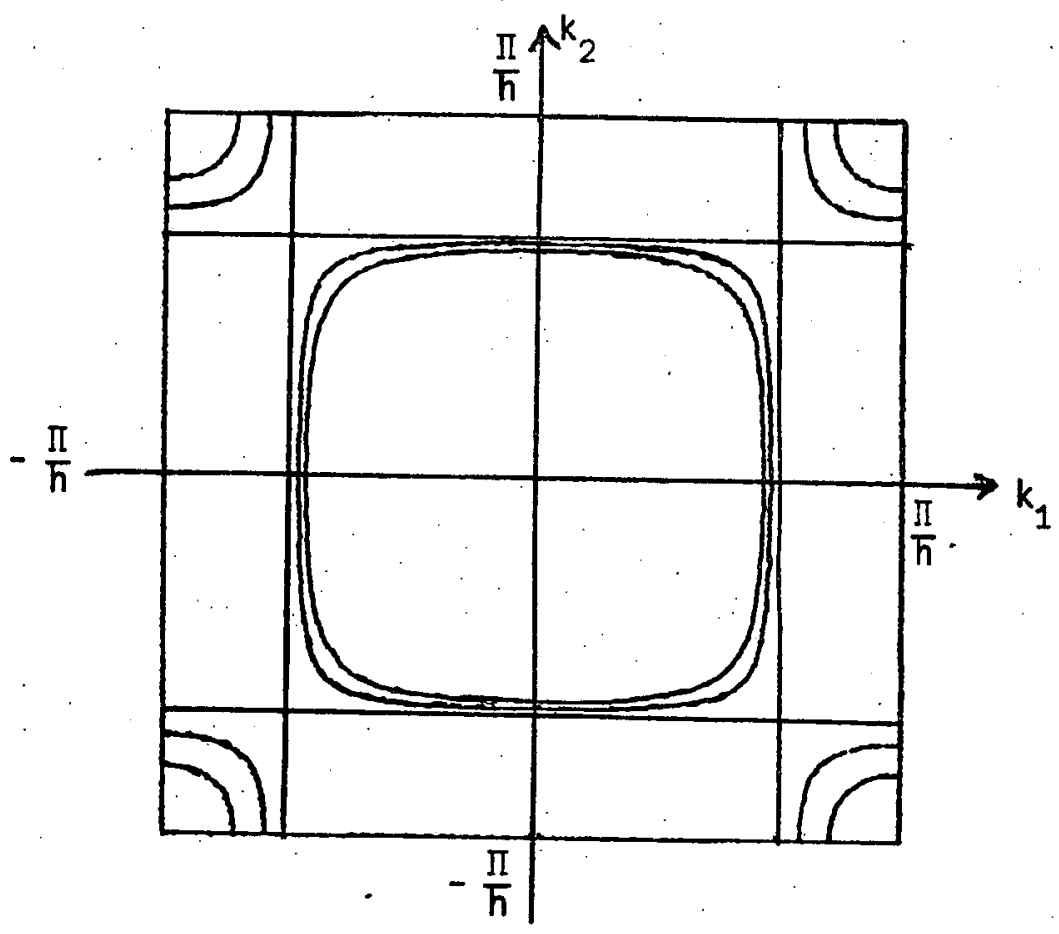

Figure 3.3

Scheme 2: The curves $\Gamma_{h}(\omega)$ for $\frac{8}{3}<\omega^{2} h^{2}<33$ 


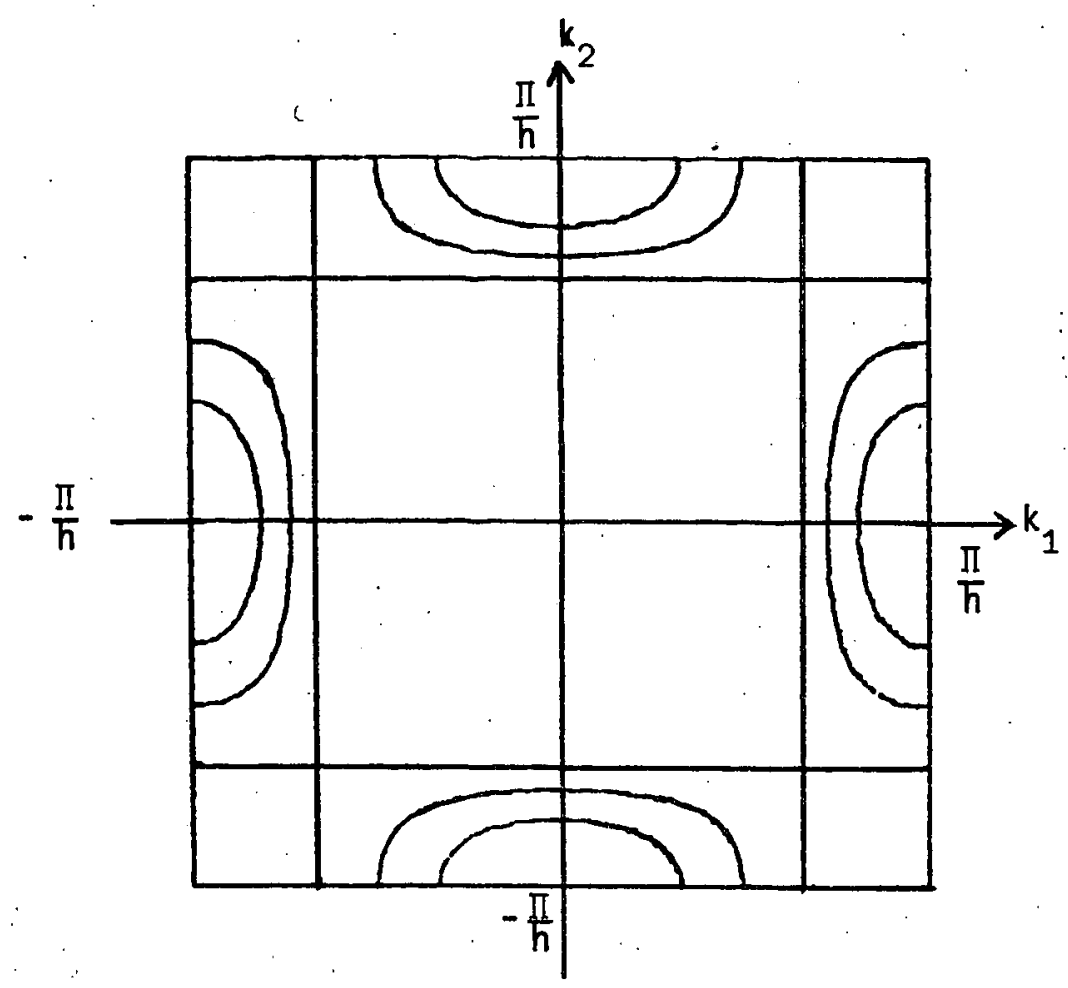

Figure 3.4

Scheme 2 : The curves $\Gamma_{h}(\omega)$ for $3<\omega^{2} h^{2}<4$

- Scheme 3

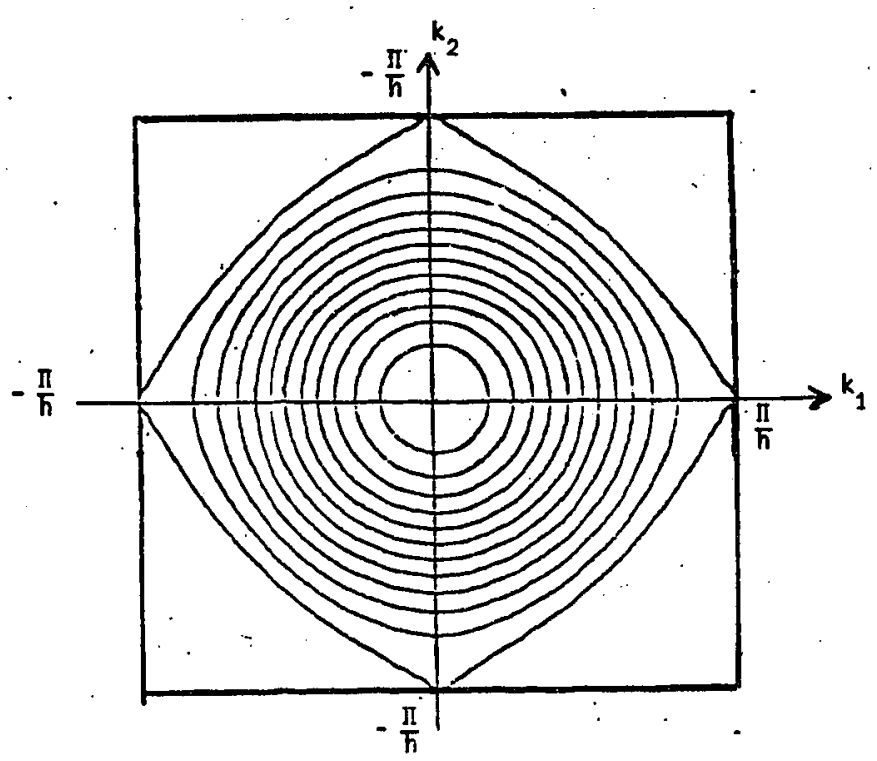

Figure 3.5

Scheme $3:$ The curves $\Gamma_{h}(\omega)$ for $0<\omega^{2} h^{2}<4$ 


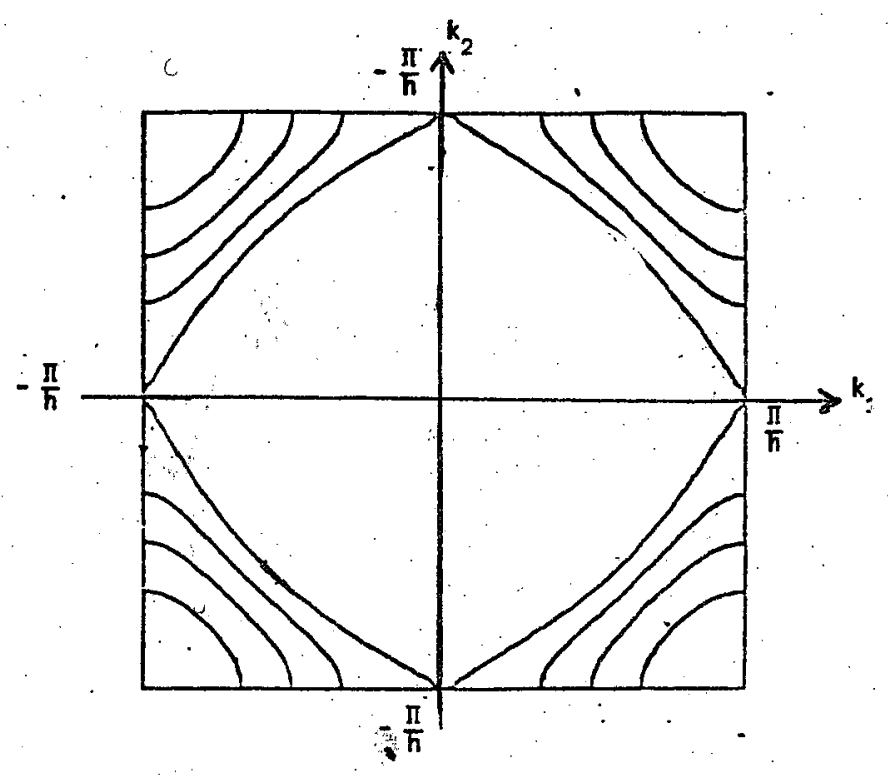

Figure 3.6

Scheme 3 : The curves $\Gamma_{h}(\omega)$ for $4<\omega^{2} h^{2}<\frac{16}{3}$

\section{III.2 - Description and comparison of the results}

In order to compare the various properties of the schemes, we must distinguish two cases. The first one corresponds to small values of wh and the second one to large ones. More precisely, in the first case, the values of wh we consider correspond to a number of mesh points per wavelength greater than five. When wh is small, the asymptotic behaviour of each discrete outgoing Green's function is very close to that of the outgoing Green's function for the continuous case. The main differences between the schemes come from the asymptotic wave fronts and the asymptotic amplitude diagrams. When we use the schemes under bad conditions, i.e. when the value of wh corresponds to a number of points per wavelength smaller than 5, the asymptotic behaviour of each discrete outgoing solution is no longer close to the one for the continuous case. Purely numerical parasitic phenomena can appear as will be described later. 


\section{III.2.1 - Small wh}

In this case, the set $g_{h}(\omega, \theta)$ (cf. section. II.2) is reduced to one element. Therefore, the asymptotic behaviour of the outgoing Green function only contains one term and we have:

$$
G_{h}\left(\omega^{2}+i 0, r, \theta\right)=G_{h}^{\infty}\left(\omega^{2}+i 0, r, \theta\right)+o\left(\frac{1}{r^{3 / 2}}\right)
$$

where the asymptotic wave

$$
e^{-i \omega t} G_{h}^{\infty}\left(\omega^{2}+i \cdot 0, r, \dot{\theta}\right)
$$

is equal to :

$$
e^{-j \omega t} G_{h}^{\infty}\left(\omega^{2}+j 0, r, \theta\right)=\frac{i}{(2 \pi)^{1 / 2}} \frac{A_{h}(\omega, \theta)}{r^{1 / 2}} e^{-j \omega\left(t-r / c_{\infty}^{h}(\omega, \theta)\right)}
$$

where $c_{h}^{\infty}(\omega, \theta)$ and $A_{h}(\omega, \theta)$ are respectively given by

The dispersion (resp. the anisotropy) of the scheme can be characterized by the variation of $c_{\infty}^{h}(\omega, \theta)$ with respect to wh (resp. $\theta$ ). Both dispersion and anisotropy can be directly seen from the wave fronts. Remark that $c_{\infty}^{h}(\omega, \theta)$ is not the phase or the group velocity of a numerical plane wave propagating in the direction $\theta$; Thus our analysis of dispersion and anisotropy is different from the one using plane waves.

Furthermore this analysis gives another way for describing the anisotropy of the scheme. In the continuous case the asymptotic amplitude decays as $1 / r^{\frac{1}{2}}$ for large $r$ uniformly with respect to $\theta$. In the discrete case, this decay is no longer uniform with $\theta$. Thus the asymptotic amplitude diagrams characterize the anisotropy of the schemes too.

Let us give the discrete asymptotic wave fronts for each scheme when wh is sufficiently small. We shall denote by $N$ the number of points per wavelength $\lambda=\frac{2 \pi}{\omega}(c=1)$, i.e. 


$$
\stackrel{0}{0}
$$




$$
\underline{10}
$$




$$
0
$$


For each scheme the result is very good when $N$ is greater than twenty. From figures 3.7-3.9 it is clear that the main phenomenon is the dispersion. The anisotropy can be considered as a "second order perburbation".

The dispersion of the three schemes is the same when we look only at the mesh directions. Therefore, in order to compare these schemes, we must study their anisotropy more carefully. The dispersion of scheme 1 is most important in the diagonal directions. For scheme 2, it is the opposite. The dispersion of scheme 3 is almost uniform with respect to $\theta$, which means that, as we could expect, the anisotropy for this scheme is very small. Indeed, as far as the anisotropy of the schemes is concerned schemes 1 and 2 have opposite properties. Then, we can expect that a convex combination of them would give a scheme whose anisotropy would be less important. It can be easily checked that :

$$
(-\Delta h)_{3}=\frac{1}{2}\left\{(-\Delta h)_{1}+(-\Delta h)_{2}\right\}
$$

In fact, $(-\Delta h)_{3}$ is the best choice for reducing anisotropy because it is the only convex sum of $(-\Delta k)_{1}$ and $(-\Delta h)_{2}$ for which the truncation error $\left|D_{h}(k)-\right| k|2|$ depends only on $|k|$ at the first order (cf. 2.3).

Now, we look at the discrete asymptotic amplitude curves for $N=5,10,20$. 


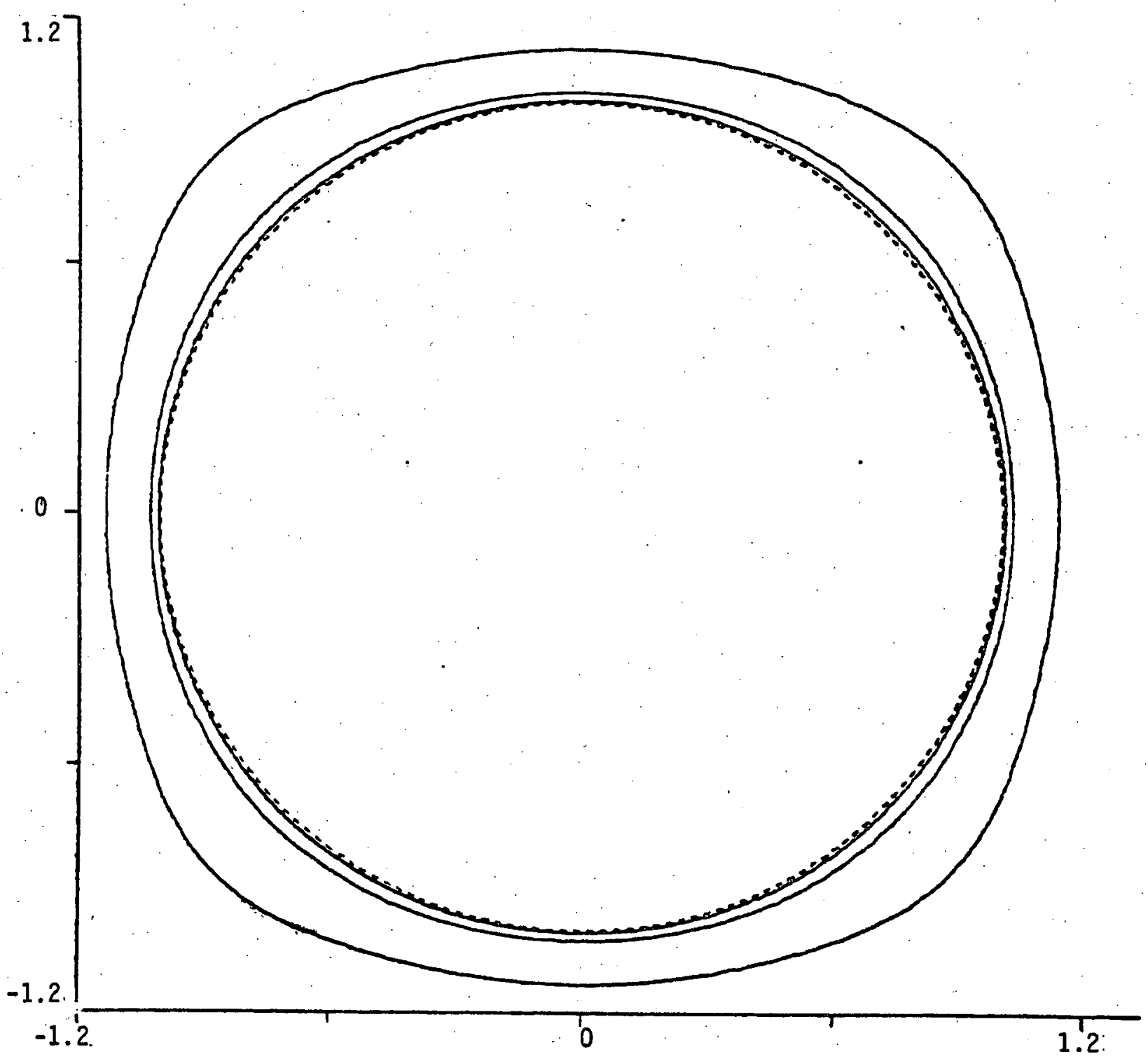

Figure 3.10

Scheme $1:$ Asymptotic Amplitude diagrams for $N=20,10,5$. 


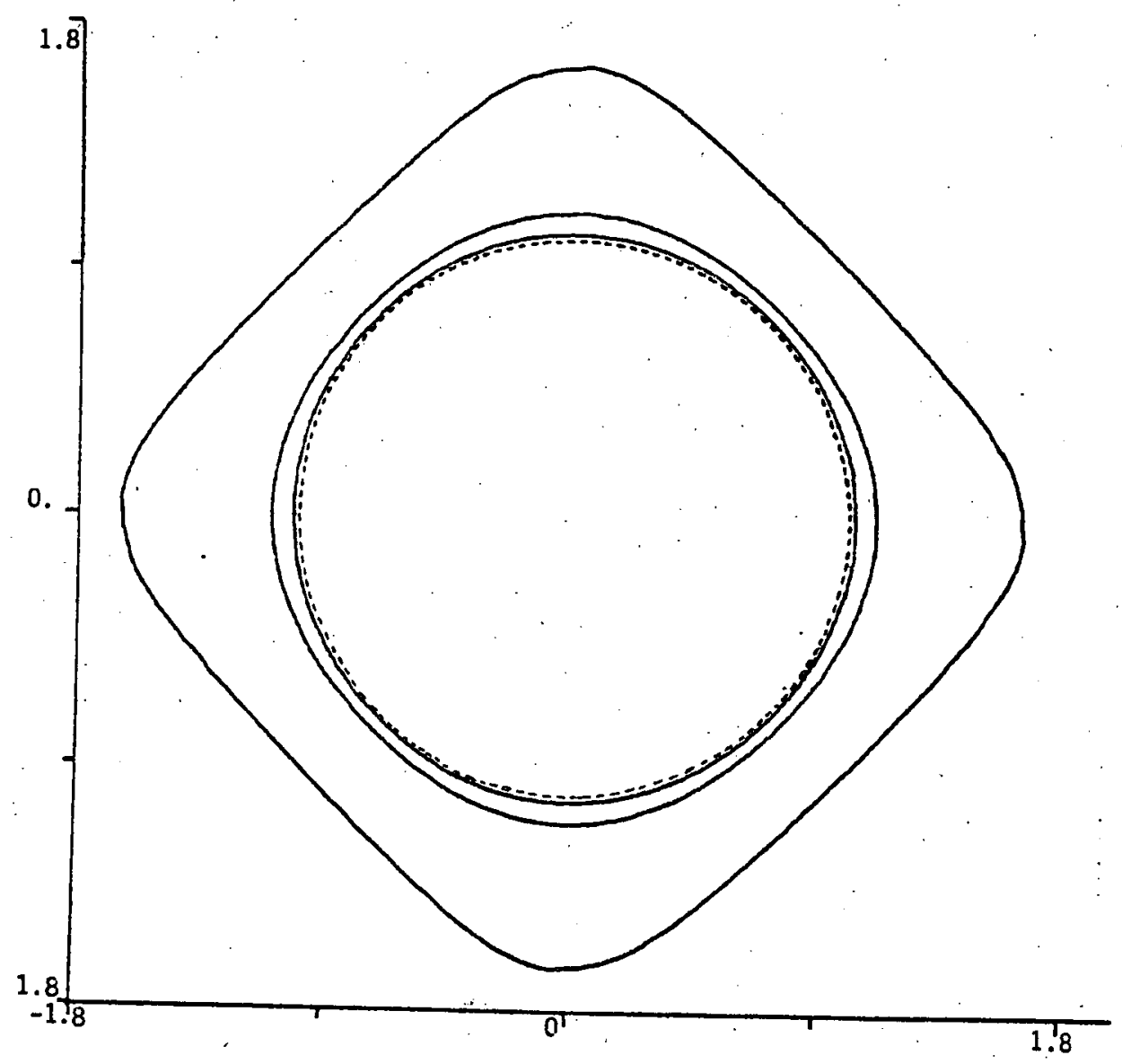

Figure 3.11

Scheme 2: Asymptotic Amplitude diagrams for $N=20,10,5$. 


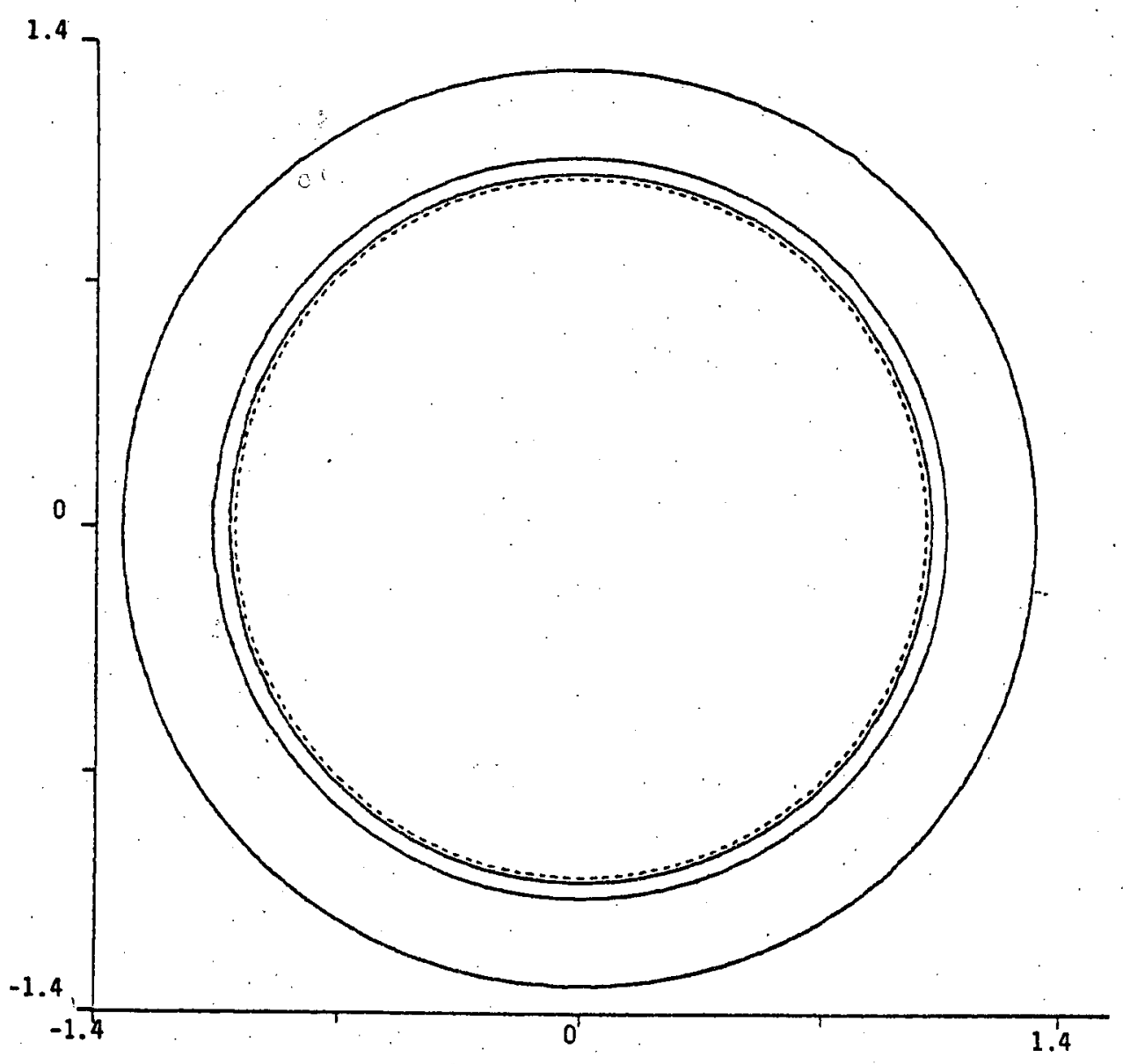

Figure 3.12

Scheme 3 : Asymptotic Amplitude diagrams for $N=20,10,5$.

The conclusions we have deduced from a careful study of the discrete asymptotic wave fronts remain the same when we study the asymptotic amplitudes.

Just remark that the difference between the discrete asymptotic amplitude and the continuous asymptotic amplitude for scheme 2 is larger than for scheme 3 , for which it is larger than for scheme 1 .

Thus, with regard to the approximation of a spherical wave associated to a Dirac source, scheme 3 is better than scheme 1 which is itseif better than scheme 2. This conclusion is deduced from the comparison of the different asymptotic diagrams. Nevertheless, if we are more interested in getting a good approximation for the amplitude of the solution the scheme 1 gives more precise results than those obtained with scheme 3 . 
III.2.2 - Large wh

In this case the comparison between the schemes becomes more difficult because the results are highly distinct.

Our study has pointed out the existence, for each scheme, of a critical value $\omega_{c}$ for the pulsation $\omega$. The results are very different depending on whether $\omega$ is greater than $\omega_{c}$ or smaller than $\omega_{c}$.

$$
\begin{array}{ll}
\text { For scheme \#1 } & \omega_{c}=\frac{2}{h} \\
\text { For Scheme \#2 } & \omega_{c}=\frac{2 \sqrt{2}}{h \sqrt{3}} \\
\text { For scheme \#3 } & \omega_{c}=\frac{2}{h} .
\end{array}
$$

When $\omega<\omega_{c}$ there always exists a unique asymptotic wave front as in the continuous case but when $\omega$ tends to $\omega_{c}$ both asymptotic wave fronts and asymptotic amplitudes are highly disturbed. For example, for scheme \#1, the asymptotic wave front tends to a square whose sides are parallel to the mesh directions (see Figure .) and the asymptotic curves are no longer convex.Moreover the asymptotic amplitudes badly behave when we look at the diagonal directions of the grid. Nevertheless it is important to note that the discrete asymptotic wave is always outgoing because of the orientation of the vectors $\nabla D_{h}(k)$ for $k$ belonging to the curve $\Gamma_{h}(\omega)$. 


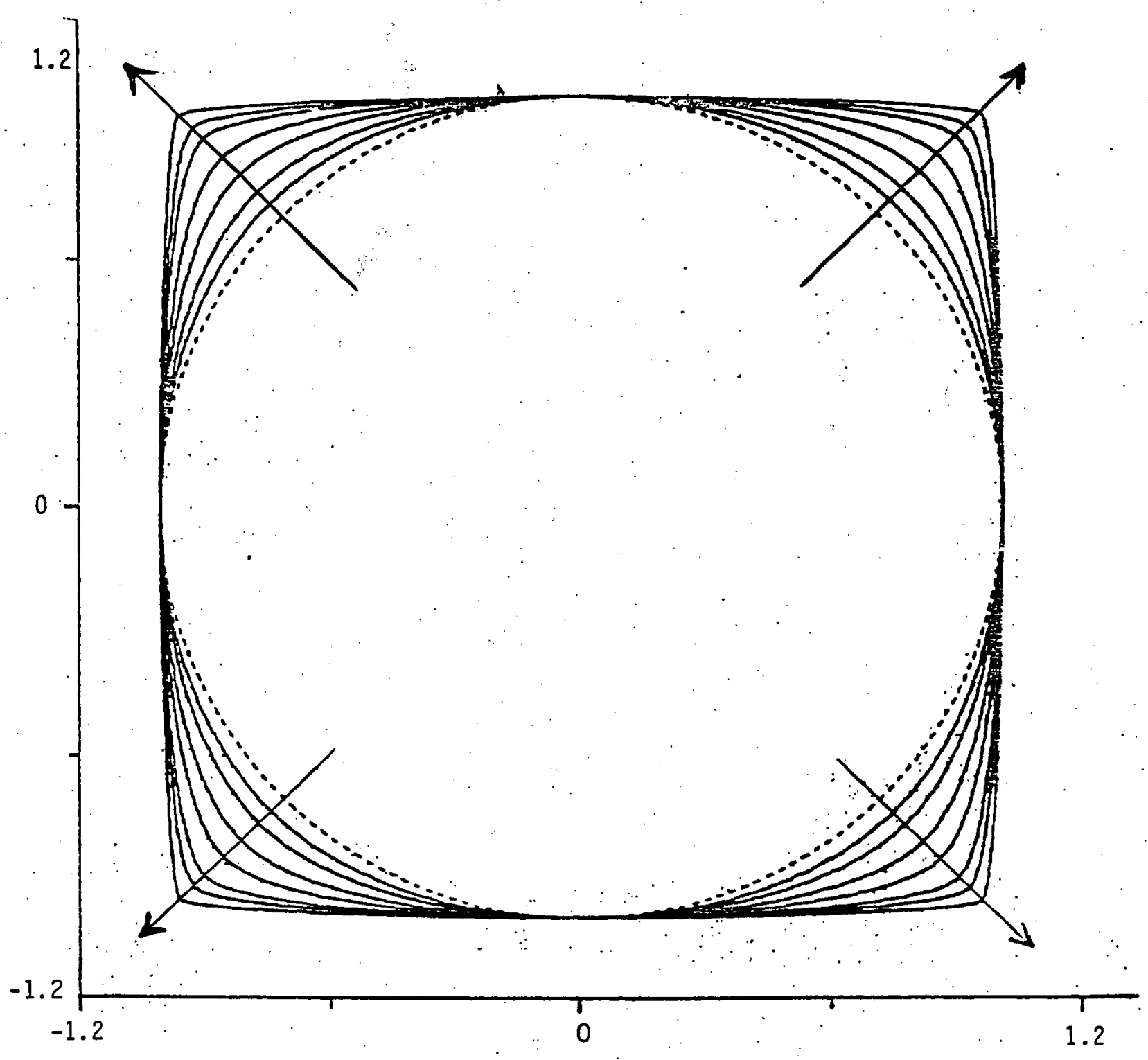

Figure 3.13

Scheme 1: Asymptotic wave fronts for $N=4,11 \%, 3,8,3.6,3.4,3.2$, $3.18,3.17,3.16$. 


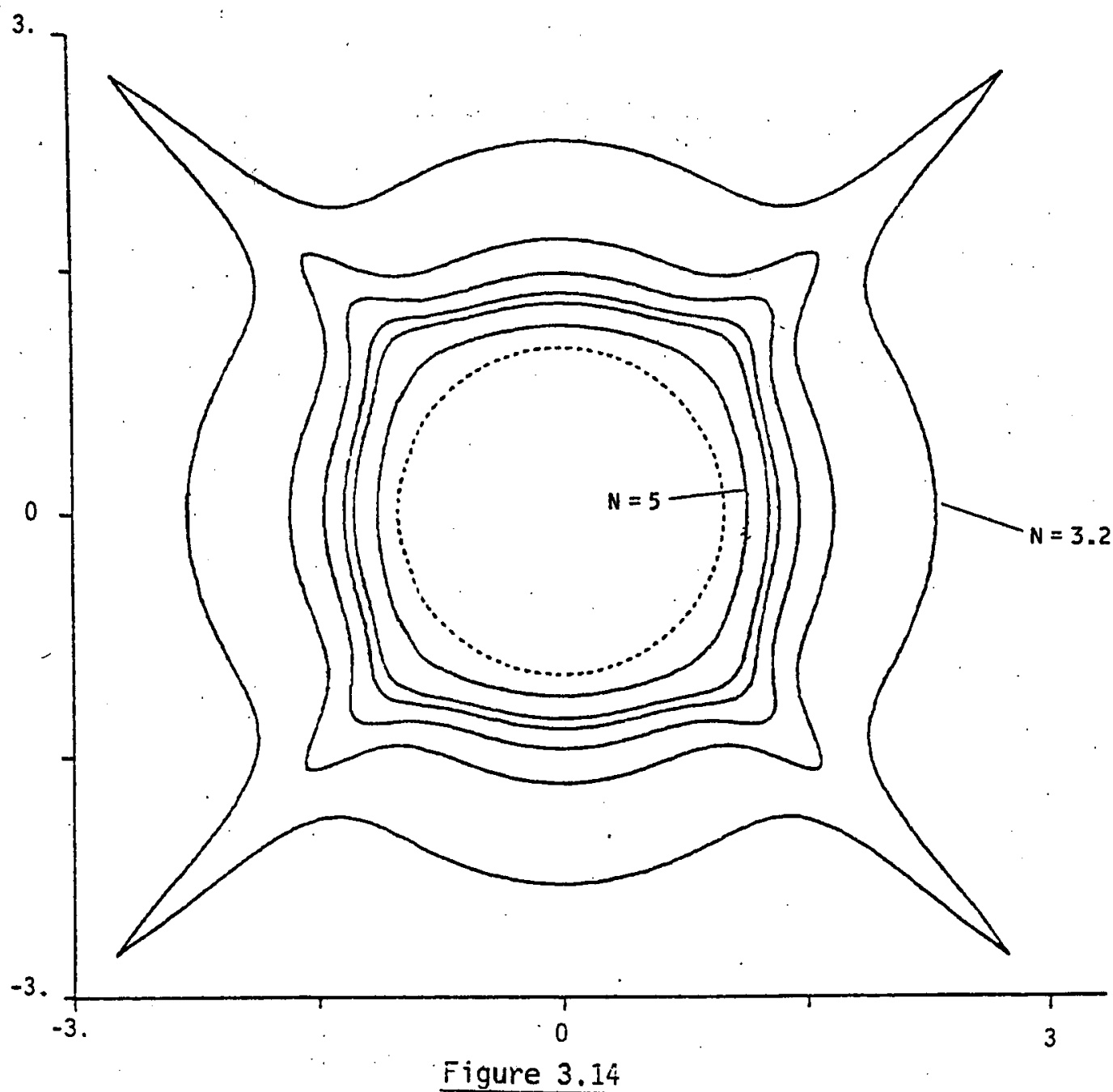

Asymptotic amplitude diagram $N=5,4,3.8,3.6,3.4 ; 3.2$.

When $\omega$ passes the critical value $\omega_{c}$, the asymptotic behaviour of the discrete solution changes drastically. More precisely for $\omega<\omega_{C}$ several purely numerical parasitic phenomena can appear and then the behaviour of the discrete solution has nothing to do with the continuous one. The nature of the parasitic phenomena really depends on the scheme one considers. For example, in the case of scheme 1 , the asymptotic wave becomes "incoming" because the orientation of the unit outgoing normal vector to $\Gamma_{h}(\omega)$ changes. In the case of the scheme 2 , the curves $\Gamma_{h}(\omega)$ are no longer simply connected. Consequently the asymptotic development of the solution contains several terms and there exist several asymptotic wave fronts, some of them asymptotic branches. In the case of scheme 3 , the curves $\Gamma_{h}(\omega)$ are no longer convex and 
therefore the asymptotic wave fronts can have several branches in particular directions:

All these parasitic phenomena are analogous to those which occur in cristallography (see [18]). Their existence is due to the geometrical properties of the curves $\Gamma_{h}(\omega)$ They are related to the fact that the function $D_{h}(k)$ is not, in the discrete case, a homogeneous function of $k$.

For each scheme, there is another critical value $\omega^{\star}$ for $\omega$ which is the upper bound of the spectrum of the operator $-\Delta_{h}$ :

$$
\begin{aligned}
& \text { For scheme \#1 } \quad \omega^{*}=\frac{2 \sqrt{2}}{h} \\
& \text { For scheme \#2 } \omega^{\star}=\frac{2}{h} \\
& \text { For scheme \#3 } \omega^{\star}=\frac{4}{h \sqrt{3}}
\end{aligned}
$$

When $\omega$ is greater than $\omega^{*}$, the outgoing Green's function is exponentially decreasing with respect to $r$ (see [16] for the details).

This study for large values of wh is not purely academic. Indeed, in real experiments, all wavelengths occur and the parasitic phenomena exist. However a careful choice of the discretization step $h$ is sufficient to reduce them.

\section{III.3 - Generalization of the results}

of course the method of analysis we have followed here can be used for studying more general. schemes. The results we have obtained for the small values of wh, mainly the existence of a unique asymptotic wave approximating the continuous one, are clearly consequences of the consistency of the schemes. Therefore we can expect to generalize these 
results for a reasonable class of numerical approximations to the Helmholtz equations. In the same way, the convergence of $G_{h}^{\infty}($.$) to G^{\infty}($. when $h$, tends to 0 can probably be studied. But our study shows that it would be difficult to give general statements for large values of wh. 


\section{REFERENCES}

[1] R.M. ALFORD, R. KELLY, U.M. BOORE, Accuracy of finite difference modeling of the acoustic wave equation, Geophysics, Vol. $39, N^{\circ} 6$, Décembre 1974, p. 834-842.

[2] A. BAMBERGER, G. CHAVENT, P. LAILLY, Etude de schémas numériques des équations de l'élastodynamique linéaire, Rapport INRIA, $\mathrm{N}^{\circ} 41$, Octobre 1980.

[3] T. BELYTSCHKO, R. MULLEN, Dispersion analysis of finite element semi-discretizations of the two-dimensional wave equation, Int. $\mathrm{J}$. for Num. Methods in Eng., Vol. 18, 1982, p. 11-19.

[4] L. TREFETHEN, Group velocity in finite difference schemes, SIAM Review, Vol. 24, No 2, Avril 1982.

L. NICOLETIS, "Simulation, numérique de Za propagation d'ondes sismiques" Thèse de Docteur Ingēnieur-Universitê P. et M. CURIE (Paris VI) 1981.

R. VICHNEVETSKY and J.B. BOWLES, Fourier Analysi.s of Numerical Approximations of Hyperbolic Equations.' SIAM Studies Applied Mathematics, Philadelphia, 1982.

[7] B.A. AULD, Acoustic fields and waves in solids I and II, Wiley Interscience, 1973.

[8] D.M. EIDUS The principle of limiting amplitude, Russion Math. Surveys 24 (1969) 91-167.

[9] V.V. GRUSIN, on Sommerfeld conditions for a certain class of partial differential equations, A.M.S., Trans., Ser. 2, 51, 1966, p. 82-112.

[10] W: LITTMAN, Fourier transforms of surface carried measures and differentiability of surface averages, Bull. American Math. Society, 69,1963 , p. $766-770$.

[11] W. LITTMAN, Decay at infinity of solutions of partial differential equations with constant coefficients, Trans. Amer. Math. Soc., 123,1966 , p. 449-454.

[12] M.MATSUMURA, Asymptotic behavior at infinity for Green's function of first order systems with characteristic of non uniform miltiplicity, Pub. R.I.M.S., Kyoto Univ., Ser. 1, 12, 1976, p. 31/-377.

[13] B.R. WAINBERG, Hypoezliptic equations in the whole space and the principle of limiting absorption, Soviet Math. Dok1., b, 1964? p. 321-324.

[14] C.H. WILCOX, Steady state wave propagation in homogeneous anisotropic media, Arch. Nat. Mech. and Ana 1., 25, 1967, p. 201-242.

[15] C.H. WILCOX, Asymptotic wave functions and energy distributions in strongly propagative anisotropicmedia, J. Math. Pures et Appliquées,
57,1978, p. 275-321. 
[16] A. BAMBERGER, J.C. GUILLOT et P. JOLY, Diffraction numérique par un mailzage réguizier. Rapport du Centre Mathêmatiques Appliquées de I'Ecole Polytechnique. Janvier 1984.

[17] L. HORMANDER, The Analysis of Iinear Partial Differental Operations $I_{\text {g }}$ Springer 1983.

[18] M. MUSGRAVE, Crystal acoustics - Introduction to the study of elastic waves and vibrations in crystals, Holden-Day, 1970 . 
